\title{
PERIODIC MAPS WHICH PRESERVE A COMPLEX STRUCTURE
}

\author{
BY P. E. CONNER AND E. E. FLOYD
}

Communicated March 2, 1964

1. Introduction. A weakly complex structure for a differentiable manifold $M$ is, roughly, a structure for the stable tangent bundle of $M$ as a complex vector space bundle; a map $T: M \rightarrow M$ is weakly complex if the differential $d T$ is stably complex linear. We consider weakly complex maps $T: M \rightarrow M$, periodic of period $p$ (usually $p$ is prime). We study such problems as the relationship between $M, F$ and the normal bundle to $F$.

There are two basic technical tools. First we study the complex bordism groups $\mathcal{u}_{n}(X)$ of a space $X$, as a generalized homology theory. For $B_{Z_{p}}$ a classifying space for the group $Z_{p}, \mathcal{U}_{n}\left(B_{Z_{p}}\right)$ is identified with the bordism group of weakly complex maps $T: M \rightarrow M$ of prime period $p$, operating on a closed manifold $M$ without fixed points. The second technical tool is the theory of $G$-bundles $E \rightarrow B$ where a compact Lie group $H$ acts on $E$ as a group of bundle maps.

This work is a supplement to our previous study of periodic maps [1], and the methods are a continuation of those. A sample of the results here were given in our Seattle lectures [2]; Zelle has also studied aspects of weakly complex actions in his thesis [6]. A full account of our results will appear later.

2. The complex bordism groups. Given a bundle $\xi$ of real $2 k$-planes over a space $X$, a complex prestructure for $\xi$ is a map $J$ mapping each fiber of $\xi$ linearly into itself and having $J^{2}=-1$. A complex structure for $\xi$ is a homotopy class of such prestructures; denote by $C(\xi)$ the set of complex structures. Denote by $k I$ the trivial $k$-plane bundle $R^{k} \times X \rightarrow X$. For $X$ a finite dimensional CW complex and for $\xi$ a bundle of real $n$-planes over $X$, a weakly complex structure for $\xi$ is an element of $C((2 k-n) I+\xi), 2 k-2 \geqq \operatorname{dim} X$; in an appropriate sense, this is independent of $k$.

A weakly complex manifold is a pair consisting of a differentiable manifold $M$ and a weakly complex structure on the tangent bundle of $M$. The boundary of a weakly complex manifold is weakly complex; each weakly complex manifold has a uniquely defined negative.

Given a pair $(X, A)$ of spaces, consider all pairs $(M, f)$ where $M$ is a weakly complex compact $n$-manifold and where $f:(M, \partial M)$ $\rightarrow(X, A)$. Two such, $\left(M_{1}, f_{1}\right)$ and $\left(M_{2}, f_{2}\right)$, are bordant if there exists 
a weakly complex compact $(n+1)$-manifold $W$ and a map $F: W \rightarrow X$ such that

(i) disjoint copies of $M_{1}$ and $-M_{2}$ are contained as smooth submanifolds of $\partial W$ with weakly complex structure induced from that of $\partial W$

(ii) $F \mid M_{i}=f_{i}$ and $F\left(\partial W-\left(M_{1} \cup M_{2}\right)\right) \subset A$. Bordism is an equivalence relation; denote the equivalence class represented by $(M, f)$ by $[M, f]_{U}$ or simply by $[M, f]$. The set $\mathfrak{u}_{n}(X, A)$ of all equivalence classes is the complex bordism group of $(X, A)$; in Milnor's notation this would be $\Omega_{n}^{U}(X, A)$, and Atiyah's notation would be $M U_{n}(X, A)$. There is $\partial: \mathfrak{u}_{n}(X, A) \rightarrow \mathfrak{u}_{n-1}(A)$ given by $\partial[M, f]=[\partial M, f \mid \partial M]$. If $\phi:(X, A) \rightarrow(Y, B), \phi_{*}: \mathfrak{u}_{n}(X, A) \rightarrow \mathfrak{U}_{n}(Y, B)$ is given by $\phi_{*}[M, f]$ $=[M, \phi f]$. Let $\mathfrak{u}_{*}(X, A)=\sum \mathfrak{u}_{n}(X, A)$.

$\left\{u_{*}(X, A), \partial, \phi_{*}\right\}$ is a generalized homology theory; that is, it satisfies the Eilenberg-Steenrod axioms except for the dimensional axiom. The coefficient group $\mathfrak{u}_{*}$ (point) is the Milnor bordism ring $\mathfrak{u}_{*}$ (in his notation, $\left.\Omega_{*}^{U}\right)$, where $\mathcal{u}_{n}$ consists of all bordism classes $[M]$ of closed weakly complex $n$-manifolds. $\mathcal{U}_{*}$ is a polynomial algebra over $Z$ with a generator in each positive dimension $2 k$.

There is the Milnor spectrum

$$
M U: \cdots, M U(k), S M U(k), M U(k+1), \cdots
$$

where $M U(k)$ is the Thom space of the universal $U(k)$-bundle, and where the map $S^{2} M U(k) \rightarrow M U(k+1)$ is given by Milnor [4]. In the following, the homology theory of a spectrum is due to G. W. Whitehead [5].

(2.1) On the category of $\mathrm{CW}$ pairs, the complex bordism homology theory is isomorphic to the homology theory of the spectrum $M U$; we have

$$
\mathfrak{u}_{n}(X, A) \cong H_{n}(X, A ; M U)=\pi_{n+2 k}(M U(k) \wedge(X / A)),
$$

\section{$k$ large.}

There is a spectral sequence $\left\{E_{p, q}^{r}\right\}$ with $E_{p, q}^{2}=H_{p}\left(X, A ; \mathfrak{u}_{q}\right)$ and whose $E^{\infty}$-term is associated with a filtration of $\mathfrak{U}_{*}(X, A)$. There is also a homomorphism $\mu: \mathfrak{u}_{n}(X, A) \rightarrow H_{n}(X, A)$ given by $\mu[M, f]=f_{*}(\sigma)$ where $\sigma \in H_{n}(M, \partial M)$ is the orientation class (a weakly complex manifold has a natural orientation).

(2.2) For $(X, A)$ a $\mathrm{CW}$ pair, the spectral sequence associated with $u_{*}(X, A)$ is trivial iff $\mu: \mathfrak{u}_{*}(X, A) \rightarrow H_{*}(X, A)$ is an epimorphism. If $H_{*}(X, A)$ has no torsion, then $\mu$ is an epimorphism. Given a set $\left\{\left[M_{i}, f_{i}\right]\right\}$ of homogeneous elements of $\mathcal{u}_{*}(X, A)$ such that $\left\{\mu\left[M_{i}, f_{i}\right]\right\}$ is a basis for $H_{*}(X, A)$, then $\mathfrak{u}_{*}(X, A)$ is a free $\mathfrak{u}_{*}$-module with base $\left\{\left[M_{i}, f_{i}\right]\right\}$. 
3. Preliminaries on weakly complex actions. Let the compact Lie group $H$ act differentiably on the compact differentiable manifold $M$; then $H$ also acts on the tangent bundle $\xi: E \rightarrow M$ via the differentials $d h, h \in H$. There is a Riemannian metric on $M$ invariant under the action of $H$; the exponential map exp: $E \rightarrow M$ is then equivariant. If $W$ is a compact smooth submanifold of $M$, invariant under $H$, then exp maps a normal cell bundle $E_{\epsilon}$ diffeomorphically and equivariantly onto a tubular neighborhood $N$ of $W$ in $M$. Hence we may identify $N$ with the cell bundle, and the action becomes an action by bundle maps. If $F=F(H, M)$ is the set of stationary points of the action, then $F$ is a finite disjoint union of smooth submanifolds of $M$; we treat it as if it were a manifold. The tubular neighborhood $N$ of $F$ is of the above type.

If $\xi: E \rightarrow M$ is the tangent bundle to $M$, then $H$ acts on the Whitney sum $(2 k-n) I+\xi$ as a group of bundle maps, acting trivially on the first coordinate. An invariant complex prestructure $J$ on $(2 k-n) I+\xi$ is a prestructure which commutes with the action of $H$. An invariant complex structure is a homotopy class of such prestructures.

A weakly complex action of the compact Lie group $H$ on the differentiable manifold $M$ is a pair consisting of a differentiable action of $H$ on $M$ and an invariant weakly complex structure for the action. We regard the stable tangent bundle $(2 k-n) I+\xi$ as a complex vector space bundle, and the differentials $d h$ as complex linear.

(3.1) Consider a weakly complex action of $H$ on $M$, where $J$ is the invariant prestructure on the stable tangent bundle $(2 k-n) I+\xi$. The restriction $(2 k-n) I+\xi_{F}$ to the stationary point set $F$ splits into the stable tangent bundle $(2 k-n) I+\xi^{\prime}$ to $F$ and the normal bundle $\eta$ to $F$ in $M$, and each is invariant under $J$. Hence $F$ is a weakly complex manifold and the normal bundle $\eta$ to $F$ is a unitary bundle, with $H$ acting on $\eta$ as a group of complex linear bundle maps.

Consider now a tubular neighborhood $N$ of the set $F$ of stationary points of a weakly complex action. On the one hand, $N$ receives an invariant weakly complex structure by restriction of that of $M$. On the other hand, $F$ is weakly complex and the normal bundle $\eta$ to $F$ is unitary. The stable tangent bundle $\xi$ of $N$ splits as the sum of the stable normal bundle $\xi^{\prime}$ to the fiber and the tangent bundle $\eta^{\prime}$ along the fiber. Now $\xi^{\prime}$ receives a weakly complex structure from $F$, and $\eta^{\prime}$ from the unitary structure of $\eta$. Hence $N$ receives a second invariant weakly complex structure. These two invariant weakly complex structures coincide. Hence the weakly complex action of $H$ on $N$ is determined completely by the weakly complex manifold $F$, the normal bundle $\eta$ to $F$ with its unitary structure, and the action of $H$ on $\eta$. 
4. Weakly complex maps of prime period. Consider a free, weakly complex action of a finite group $H$ on a closed $n$-manifold $M$; denote the pair by $(H, M)$. There is a natural equivariant bordism group of such pairs; denote the bordism class represented by $(H, M)$ by $[H, M]_{U}$ or simply by $[H, M]$. Given a pair $(H, M)$, then $M / H$ is weakly complex and $M \rightarrow M / H$ is a principal $H$-bundle. Letting $f: M / H \rightarrow B_{H}$ be a classifying map for the principal $H$-bundle, we receive an element $[M / H, f] \in \mathfrak{u}_{n}\left(B_{H}\right)$. There results an isomorphism of the bordism group of free weakly complex actions with $\mathfrak{u}_{n}\left(B_{H}\right)$; we identify the two. The $\mathfrak{u}_{*}$-module structure of $\mathfrak{u}_{*}\left(B_{H}\right)$ is given by letting $[H, M]\left[M^{\prime}\right]$ denote $\left[H, M \times M^{\prime}\right]$ where $H$ acts on $M \times M^{\prime}$ by $h(x, y)=(h x, y)$.

In the remainder of this section consider $H=Z_{p}, p$ a prime. An action of $Z_{p}$ is equivalent to a map $T: M \rightarrow M$ of period $p$; we use either $\left[Z_{p}, M\right]$ or $[T, M]$ for the element of $\mathcal{u}_{*}\left(B_{Z_{p}}\right)$.

Consider first a weakly complex map $T: M \rightarrow M$, on a closed manifold, of period $p$, where $T$ has fixed points. If $B$ is the boundary of a tubular neighborhood $N$ of the fixed point set $F$, then $T$ on $B$ gives a free action of $Z_{p}$ and $[T, B]=0$ in $\mathcal{u}_{*}\left(B_{Z_{p}}\right)$. In this way we get relations in $\mathfrak{U}_{*}\left(B_{Z_{p}}\right)$.

Let $\eta$ be the normal bundle to $F$ and let $\eta^{\prime}$ be the Whitney sum of a trivial complex line bundle and $\eta$. An action of $Z_{p}$ on the line bundle is given by multiplication by $\exp 2 \pi i / p$; the diagonal action gives an action of $Z_{p}$ on $\eta^{\prime}$. Let $B^{\prime}$ be the sphere bundle of $\eta^{\prime}$, and let $T^{\prime}: B^{\prime} \rightarrow B^{\prime}$ be the periodic map resulting from the action of $Z_{p}$ on $\eta^{\prime}$.

(4.1) If $T: M \rightarrow M$ is a weakly complex map of prime period $p$ and if $B^{\prime}$ is as above, then $\left[T^{\prime}, B^{\prime}\right]=\left[T_{1}, S^{1}\right][M]$ in $\mathcal{u}_{n+1}\left(B_{Z_{p}}\right)$, where $T_{1}(z)=(\exp 2 \pi i / p) \cdot z$.

The proof is similar to [1, Theorem 35.1$]$. We can go on to analyze $\mathcal{u}_{*}\left(B_{Z_{p}}\right)$ as in [1]. For each $S^{2 n-1}$, pick a unitary $T: S^{2 n-1} \rightarrow S^{2 n-1}$ of period $p$ and without fixed points. Then $\left\{\left[T, S^{2 n-1}\right]\right\}$ generates the

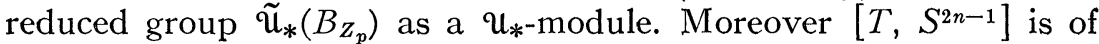
order $p^{k+1}$ where $2 k(p-1)<2 n-1<2(k+1)(p-1)$. $\left[T, S^{2 k(p-1)+1}\right]$ is of particular interest. Here $p^{k}\left[T, S^{2 k(p-1)+1}\right]=b\left[T_{1}, S^{1}\right]\left[P_{p-1}(C)\right]^{k}$, where $b \neq 0 \bmod p$. The explicit additive structure of $\mathcal{U}_{*}\left(B_{z_{p}}\right)$ is obtained.

Perhaps the most interesting feature in the complex case is the case $p=2$, which is completely different from the oriented bordism situation. Consider $\mathfrak{u}_{*}\left(B_{Z_{2}}\right)=\mathfrak{u}_{*}\left(P_{\infty}(R)\right)$, or alternatively the bordism theory of fixed point free weakly complex involutions. The generators are $\left[T, S^{2 n-1}\right]$ where $T$ is the antipodal map, $\left[T, S^{2 n-1}\right]$ is of order $2^{n}$ and $2^{n-1}\left[T, S^{2 n-1}\right]=\left[T_{1}, S^{1}\right]\left[P_{1}(C)\right]^{n-1}$. 
(4.2) Suppose that $M^{2 n}$ is a closed almost complex manifold and that $T: M^{2 n} \rightarrow M^{2 n}$ is a differentiable involution commuting with the almost complex structure. If $T$ has only isolated fixed points, then the number of fixed points is of the form $k \cdot 2^{n}$. Moreover $\left[M^{2 n}\right]=k\left[P_{1}(C)\right]^{n}$ in $\mathfrak{u}_{*} / 2 \mathcal{u}_{*}$.

As an example it is easy to construct a complex analytic involution on $\left[P_{1}(C)\right]^{n}$ having exactly $2^{n}$ fixed points.

5. Weakly complex actions of $\left(Z_{p}\right)^{k}$ and $Z_{p}{ }^{k}$. Consider a weakly complex action of $\left(Z_{p}\right)^{k}, p$ a prime; that is, consider maps $T_{i}: M \rightarrow M$, $i=1, \cdots, k$, of period $p$ which commute and all of which have invariant the same weakly complex structure on $M$. Our previous methods $[1, \S 43]$ apply to show that if $\left(Z_{p}\right)^{k}$ acts in a weakly complex fashion on the closed $n$-manifold $M$ without stationary points, then the Chern numbers of $M$ are all divisible by $p$. We strengthen this to the following.

(5.1) The ideal of $\mathfrak{U}_{*}$ consisting of all elements admitting a repre. sentative $M$ upon which there is a weakly complex action of $\left(Z_{p}\right)^{k}$ without stationary points coincides with the ideal of $\mathfrak{u}_{*}$ consisting of all elements whose Chern numbers are all divisible by $p$.

In the above, there is exhibited a sequence $p, M^{2 p-2}, \cdots$, $M^{2 p^{k}-2}, \cdots$ with $\left(Z_{p}\right)^{k+1}$ acting as required on $M^{2 p^{k}-2}$. Here $M^{2 p^{k}-2}$ is the submanifold of $P_{p^{k}}(C)$ consisting of all $\left[z_{0}, \cdots, z_{p^{k}}\right]$ with $\sum z_{i}^{p}=0$. Each generator of $\left(Z_{p}\right)^{k}$ sends $\left[z_{0}, \cdots, z_{i}, \cdots\right]$ into $\left[\rho_{0} z_{0}, \cdots, \rho_{i} z_{i}, \cdots\right]$ for appropriate choice of $p$ th roots $\rho_{i}$ of unity. The bordism classes $p,\left[M^{2 p-2}\right],\left[M^{2 p^{2}-2}\right], \cdots$ generate the ideal of elements of $\mathfrak{U}_{*}$ all of whose Chern numbers are divisible by $p$.

In a similar fashion, we can consider the ideal of the oriented bordism ring $\Omega_{*}$ represented by manifolds $M$ upon which some $\left(Z_{p}\right)^{k}$ acts, preserving the orientation and without stationary points, for $p$ an odd prime. This ideal consists of all elements having all Pontryagin numbers divisible by $p$. The analogous problem in which $k$ is fixed continues to be unsolved.

We consider now actions of $Z_{p^{k}}$, solving a problem that was only treated with partial success in our previous work $[1, \S 45]$.

(5.2) Suppose that $T: M \rightarrow M$ is a weakly complex map of prime power period $p^{k}$ on the closed manifold $M$. If $T$ has no fixed points, then $[M] \in p u_{*}$. Similarly if $T$ is a differentiable orientation preserving map of prime power period $p^{k}, p$ an odd prime, acting on the closed oriented manifold without fixed points, then $[M] \in p \Omega_{*}$.

Besides using the results of $\$ 4$, the proof uses a desingularization process based on expressing every lens space as a boundary in a specific way. Namely consider a closed manifold $M$ upon which the compact Lie group $G$ acts in a weakly complex fashion. Let $S^{1} \subset G$ be in the center of $G$ and suppose $S^{1}$ has no stationary points in $M$. Then 
there exists a weakly complex action of $G$ on a compact manifold $W$ so that $\partial W=M$ and so that the action of $G$ on $W$ restricts to the action of $G$ on $M$.

For $p=2$, the conclusion of $(5.2)$ in the oriented case would be definitely false $[1, \S 45]$. However for $p$ odd the normal bundle $\eta$ to the fixed point set $F$ can be reduced to the unitary group, and the methods of the complex case apply.

6. Equivariant maps. In this section we indicate the application of bordism to nonexistence theorems for equivariant maps. For simplicity of statement, we confine ourselves to $p=2$; the results can be extended to any $p$.

(6.1) Theorem. Consider an element $\gamma \in \tilde{\mathfrak{u}}_{2 n-1}\left(B_{Z_{2}}\right)$. The order of $\gamma$ divides $2^{k}$ if and only if there exists a representative $\left(T, M^{2 n-1}\right)$ of $\gamma$ and $a \operatorname{map} f: M^{2 n-1} \rightarrow S^{2 k}$ with $f(T x)=-f(x)$ for all $x \in N^{2 n-1}$.

In a different terminology, the order of $\gamma$ divides $2^{k}$ if and only if some representative $\left(T, M^{2 n-1}\right)$ is of co-index $\leqq 2 k$. Hence it becomes of geometric interest to compute the order. We do not know any general methods; we can however make the computation in a special case. Namely, let $Z_{4}$ act in a free unitary fashion on $S^{2 n-1}$. Now $Z_{2} \subset Z_{4}$, and we obtain a free weakly complex action of $Z_{2}$ on $S^{2 n-1} / Z_{2}$ $=P_{2 n-1}(R)$. We show that the order of $\left[Z_{2}, P_{4 n+1}(R)\right]$ is $2^{n+1}$; hence by (6.1) there exists no equivariant map of $P_{4 n+1}(R)$ into $S^{2 n}$. This represents some progress on a question we have investigated previously [3]. Anderson at Berkeley has proved similar nonexistence theorems by $K$-theory. Our proof uses a natural homomorphism $\mathfrak{u}_{m}\left(B_{S^{1}}\right)$ $\rightarrow \mathfrak{U}_{m+1}\left(B_{Z_{2}}\right)$, and a study of $f_{*}: \mathfrak{U}_{*}\left(B_{S^{1}}\right) \rightarrow \mathfrak{U}_{*}\left(B_{S^{1}}\right)$ where $f$ acts on a generator $a \in H^{2}\left(B_{S^{1}}\right)$ by $f^{*}(a)=2 a$.

\section{BIBLIOGRAPHY}

1. P. E. Conner and E. E. Floyd, Differentiable periodic maps, Springer, Berlin, 1964.

2. Cobordism theories, Seattle Conference on Differential and Algebraic Topology (mimeographed), Amer. Math. Soc., Providence, R. I., 1963.

3. - Fixed point free involutions and equivariant map. II, Trans. Amer. Math. Soc. 105 (1962), 222-228.

4. John Milnor, On the cobordism ring $\Omega^{*}$ and a complex analogue, Amer. J. Math. 82 (1960), 505-521.

5. G. W. Whitehead, Generalized homology theories, Trans. Amer. Math. Soc. 102 (1962), 227-283.

6. K.-G. Zelle, Symmetrien von Mannigfaltigkeiten und Charakteristiche Zahlen, thesis, Bonn, 1963.

UNIVERSITY OF VIRGINIA AND

INSTITUTE FOR AdVANCED STUdy 